\title{
Efficiency Increase of Wet Gas Cleaning from Dispersed Admixtures by the Application of Ultrasonic Fields
}

\author{
Vladimir N. KHMELEV, Andrey V. SHALUNOV, Roman N. GOLYKH, Roman S. DOROVSKIKH, \\ Viktor A. NESTEROV, Sergey S. KHMELEV, Ksenija V. SHALUNOVA
}

Biysk Technological Institute (branch) of the AltSTU

659305, Biysk, Russia; e-mail: romang190@gmail.com

(received September 19, 2015; accepted September 21, 2016)

\begin{abstract}
The article presents the results of research aimed at increase of the efficiency of gas cleaning equipment based on the Venturi tube using high-intensity ultrasound. The model based on known laws of hydrodynamics of multiphase mediums of dust-extraction in Venturi scrubbers was proposed. Modification of this model taking into account ultrasonic field allows evaluating optimum modes (sound pressure level) and conditions (direction of ultrasonic field, square and number of ultrasonic sources) of ultrasonic influence. It is evaluated that optimum for efficient gas cleaning is the mode of ultrasonic action at the frequency of $22 \mathrm{kHz}$ with sound pressure level of $145 \ldots 155 \mathrm{~dB}$ at the installation of two radiators with area of $0.14 \mathrm{~m}^{2}$, four radiators with area of $0.11 \mathrm{~m}^{2}$ or six radiators with area of $0.08 \mathrm{~m}^{2}$ at the angle of 45 degrees to the axis of Venturi tube. Numerical calculations showed that realization of ultrasonic action is the most efficient for the reduction (up to 15 times) of the content of fine-dispersed fraction $(2 \mu \mathrm{m}$ and less), which is impossible to extract without ultrasonic action. The received theoretical results were confirmed by industrial testing by typical dust-extraction plant and used as foundations of development of apparatuses with the radiators of various sizes.
\end{abstract}

Keywords: dust extraction plant; Venturi tube; ultrasound; coagulation; dispersed particles.

\section{Introduction}

The sequence of industrial development is noticeable deterioration of atmosphere condition. The main sources of air pollution are industrial enterprises and thermal power stations (LAVELY, Ferguson, 1996; KHMElev et al., 2010b; 2010c; 2012; ST. Clair, 1949), which according to specialists' estimations, every day inject into the atmosphere up to 1 billion tons of dispersed particles with the size of $1 . \ldots 200 \mu \mathrm{m}$. The finest of them (less than $5 \mu \mathrm{m}$ ) are especially dangerous for people health, flora and fauna (KHMELEV et al., 2010b; 2010c; 2012; St. Clair, 1949; Mohamed, 2011) as they easily penetrate to lung alveolus and can be carriers of viruses and bacteria causing various diseases.

Main efforts of the designers of gas cleaning equipment are focused on the solution of the problem of dust particle catching.

For the purification of output gases from dispersed admixtures (ShTOKMAn, 1977; 1999; Uzhov, 1981; Balabekov, Baltabaev, 1991; Kropp, Akbrut, 1977) technical devices based on the use of different physical actions are applied. However, efficiency of all used devices is very low at collecting of particles of less than $5 \mu \mathrm{m}$. The attempts of dry aggregation of such particles by external energy action (for instance, by the application of ultrasonic vibrations) do not take required effect due to instability of formed aggregates in air flows.

Thus, to increase efficiency of fine-dispersed particles, water aerosol providing preliminary absorption of solid particles for further inertial collecting by the drop catcher is used. Such approach allows increasing efficiency of collecting in comparison to dry dust catching (Shtokman, 1977; 1999; Uzhov, 1981; BalaBeKOV, BALTABAEv, 1991). It is performed in industrial Venturi scrubbers (tubes) (LAVELy, Ferguson, 1996; Kropp, AkBrut, 1977) which are characterized by maximum efficiency of industrial gas cleaning providing residual dust content of about $0.5 \mathrm{~g} / \mathrm{m}^{3}$ at the output (KROPP, AKBrUT, 1977). Unfortunately, even wet dust collecting does not meet the requirements of modern ecological standards of maximum permissible concentration of polluting emissions (no more than $0.2 \mathrm{~g} / \mathrm{m}^{3}$ (Hygienic Standards 2.1.6.695-98, 1998)). 
It is known that action of ultrasonic vibrations contributes to aggregation of particles for further collection by the standard gas cleaning equipment. The analysis of ultrasonic coagulation possibilities (GALLEGOJuAres et al., 1999; Shalimo, 1965; Danser, NeuMANN, 1949; KHMELEV et al. 2008) allows to suggest that efficiency of small solid particles aggregation with large water drops should be in proportion to the level of sound pressure generated by ultrasonic vibrations in Venturi tube (Kouzov et al., 1993). As there is no equipment for ultrasonic coagulation in Venturi tubes at present, it is impossible to prove efficiency of ultrasonic action for the solution of gas cleaning problems.

The aim of the paper is development and study of the ultrasonic equipment, which allows realizing maximum efficient modes and conditions of ultrasonic action (frequency, intensity, size of the zone and time of action) for efficiency increase of collection of finedispersed particles in Venturi tubes.

For development and application of the equipment it is necessary to provide maximum efficient introduction of ultrasonic vibrations to the volume of dust collecting device without changes of dust and gas flow conditions, i.e. without disturbance of functional possibilities of existing apparatuses. As applied gas cleaning devices vary in sizes, forms, methods of spraying by liquid drops, modes of movement and parameters of collected dust (sizes of particles and concentration), there is a need to formulate the basic principles of highefficient ultrasonic action realized at any devices of industrial dust collecting.

In order to achieve stated aim it is necessary to solve sequentially following tasks:

- to develop the model of dust collection in Venturi scrubbers which allows to analyze the interaction process of solid particles with water drops with or without external energy action,

- on the base of model analysis to study the process for the definition of dependences of gas cleaning efficiency on the modes (frequency and intensity of vibrations) and conditions (direction, size of the zone, uniformity, time) of ultrasonic action;

- to determine conditions of introduction and number of the radiators for maximum efficiency of ultrasonic action and for design of required ultrasonic radiators.

This article is devoted to the solution of the above stated tasks.

\section{Dust collection model in Venturi scrubbers}

To determine modes and conditions of ultrasonic action providing maximum efficiency of wet gas cleaning it is necessary to study the interaction process of solid particles with water drops in hydrodynamic conditions of gas flow performed in industrial Venturi scrubbers (Kouzov et al., 1993).

For the analysis of particles interaction and efficiency rating of wet gas cleaning the model of dust and gas flow in the system "Venturi tube-drop catcher" based on independent consideration of gas phase flow, motion and absorption of dispersed particles is proposed. Such study is possible as volume concentration of solid particles in industrial waste gases does not exceed $10^{-4}$ (less than $100 \mathrm{~g} / \mathrm{m}^{3}$ ) (KHMELEV et al., 2010b; 2010c; 2012; St. Clair, 1949; Mohamed, 2011).

In proposed model gas phase flow is described by the system of Navier-Stokes equations (1), (2), state of gas medium (3) and heat conductivity (4) on the assumption that gas flow is stationary and laminar:

$$
\begin{aligned}
\operatorname{div}(\rho \mathbf{v}) & =0, \\
\rho(\mathbf{v}, \nabla) \mathbf{v} & =-\nabla p, \\
p M & =\rho R T, \\
(\mathbf{v}, \nabla) T & =\chi \Delta T,
\end{aligned}
$$

where $\rho$ is the density of gas flow, $\mathrm{kg} / \mathrm{m}^{3} ; \mathbf{v}$ is the rate of gas flow, $\mathrm{m} / \mathrm{s} ; p$ is the barometric gas pressure, $\mathrm{Pa}$; $T$ is the temperature of gas flow, $\mathrm{K} ; R$ is the universal gas constant equal to $8.31 \mathrm{~J} /($ mole $\cdot \mathrm{K}) ; M$ is the molar mass of gas, $\mathrm{kg} / \mathrm{mole} ; \chi$ is the temperature conductivity coefficient, $\mathrm{m}^{2} / \mathrm{s}$.

The modelling of gas flow was carried out at boundary condition of slipping (FlaGAN, SEInfELd, 1988) near the walls of Venturi tube.

The field of gas velocities calculated on the base of the Eqs. (1)-(4) by finite element method is the input for modelling of motion and absorption of dispersed phase. Modelling of dispersed phase motion is based on Lagrange approach. It is realized by assignment of coordinate values to each particle in a current moment of time. Further motion of each particle is traced individually depending on disturbing force of streamlining from the side of the main gas flow and variable force (ultrasonic action). It should be noted, that in real conditions the number of particles located in the apparatus at a time can exceed $10^{10}$ (if dust content of gas flow at the input is more than $15 \mathrm{~g} / \mathrm{Nm}^{3}$, sizes of the particles are $2 . .90 \mu \mathrm{m}$ and the sizes of Venturi tube are more than $\varnothing 1.3 \times 10 \mathrm{~m}$ ). That is why each group of fixed number of the particles (normally $10^{4} \ldots 10^{6}$ ) positioned in some local area of small volume is replaced by one particle having equal physical properties (diameter, mass, velocity, trajectory, etc.) of all group (Flagan, SEInfeld, 1988). It provides reduction of calculation time of dispersed phase motion in several times. The motion of each particle is described by Newton law, according to the law its velocity is changed due to the action of certain forces aggregate (Flagan, Seinfeld, 1988; Sommerfeld, 2001) from 
the side of gas phase molecules. The equation is used on assumption about sphere shape of particles.

$$
\begin{aligned}
m_{\mathrm{p}} \frac{\partial \mathbf{v}_{\mathrm{p}}}{\partial t}= & 3 \pi \mu d C_{\mathrm{cor}}\left(\mathbf{v}_{\mathrm{f}}-\mathbf{v}_{\mathrm{p}}\right) \\
& +\frac{\pi d^{3} \rho_{\mathrm{f}}}{12}\left(\frac{\partial \mathbf{v}_{\mathrm{f}}}{\partial t}-\frac{\partial \mathbf{v}_{\mathrm{p}}}{\partial t}\right)
\end{aligned}
$$

where $m_{\mathrm{p}}$ is the mass of the particle, $\mathrm{kg} ; d$ is the diameter of the particle, $\mathrm{m} ; \mathbf{v}_{\mathrm{f}}$ is the gas velocity calculated from Eqs. (1)-(4), $\mathrm{m} ; \mathbf{v}_{\mathrm{p}}$ is the particle velocity, $\mathrm{m} ; \mu$ is the dynamic substance viscosity of the basic phase, $\mathrm{Pa} \cdot \mathrm{s} ; C_{\text {cor }}$ is its coefficient of viscous resistance.

Index $p$ (particle) is related to the particle while in$\operatorname{dex} f$ (fluid) is related to the substance of basic phase.

For the description of the particle behaviour the following forces acting on it are assigned:

- Stokes force of viscous streamline $3 \pi \mu d C_{\text {cor }}$ $\left(\mathbf{v}_{\mathrm{f}}-\mathbf{v}_{\mathrm{p}}\right.$ ) (FlaGan, SEInfeld, 1988);

- force of pressure differential due to the acceleration of basic phase flow defined by known expression $\frac{\pi d^{3} \rho_{\mathrm{f}}}{12}\left(\frac{\partial \mathbf{v}_{\mathrm{f}}}{\partial t}-\frac{\partial \mathbf{v}_{\mathrm{p}}}{\partial t}\right)$ (FLAGAN, SEINFELD, 1988).

The calculation of reciprocal collision of the dispersed particles pairs due to difference of velocities is carried out with the help of probabilistic approach, according to which the number of solid particle (ash) collisions with water drops in the volume $V$ is defined by the following expression (5) (SOMMERFELD, 2001)

$$
N=0.25 n_{1} n_{2} \pi\left(d_{1}+d_{2}\right)^{2} v_{\text {rel }} V \Delta t,
$$

where $n_{1}, n_{2}$ is the concentration of solid particles and water, $\mathrm{m}^{-3} ; d_{1}, d_{2}$ is the conventional diameter of solid particles and water, $\mathrm{m}$; $v_{\text {rel }}$ is the relative velocity of motion of solid particle and water, $\mathrm{m} / \mathrm{s} ; \Delta t$ is the period of time, s.

It is assumed, that as a result of collision of two dispersed particles new particle having conventional diameter $\sqrt[3]{d_{1}^{3}+d_{2}^{3}}$ is formed.

Because of high velocity of gas flow in Venturi tube of $15-60 \mathrm{~m} / \mathrm{s}$ (KROPP, AKBrut, 1977) the assumption about inelastic reflection of particles is true. That is why it is proposed to supplement the equation of particle motion (5) with additional boundary condition on the wall of the gas cleaning apparatus, located in the following, when the particle hits the wall, the tangent component of particle velocity remains, but normal component becomes close to zero (Fig. 1). The boundary condition is caused by wetness of the Venturi tube wall.

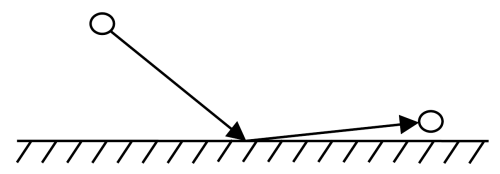

Fig. 1. Scheme of inelastic reflection of the particle from Venturi tube.
The solution of motion equation of gas flow (1)(4) and particles (5) allows to calculate dust extraction plant efficiency. Generally, the efficiency is defined as a ratio between input dust total mass per a second $C_{\text {in }}(\mathrm{kg} / \mathrm{s})$ and output dust total mass per a second $C_{\text {out }}(\mathrm{kg} / \mathrm{s})$ (ShilyaEv et al., 2006),

$$
n=\left(1-\frac{C_{\text {in }}}{C_{\text {out }}}\right) \cdot 100 \% \text {. }
$$

$C_{\text {in }}$ is defined as $\alpha G$, where $\alpha$ is a dust content at extraction plant input $\left(\mathrm{kg} / \mathrm{m}^{3}\right), G$ is gas flow rate $\left(\mathrm{m}^{3} / \mathrm{s}\right)$. To define $C_{\text {out }}$, the calculation of particles trajectory is performed taking into account their agglomeration. Then the nominal sizes $d_{\mathrm{k}}$ and number $N(\Delta \underline{t})$ of particles passing through the section of the output pipe of gas cleaning plant in time $\Delta t(\mathrm{~s})$ are fixed. Because the particles ejected from dust extraction plant contain fly ash and water drop, to determine $C_{\text {out }}$ for fly ash dust the indicator function of particle type is used.

$$
H_{d_{\text {min }}}\left(d_{\mathrm{k}}\right)=\left\{\begin{array}{l}
1 ; d_{\mathrm{k}}<d_{\min }, \\
0 ; d_{\mathrm{k}} \geq d_{\min },
\end{array}\right.
$$

where $d_{\text {min }}$ is minimum diameter of water drop, $\mathrm{m} ; d_{\mathrm{k}}$ is diameter of particle passing through the section of the output pipe of gas cleaning plant, $\mathrm{m}$.

This function equals 1 , if output particle is fly ash (diameter less than minimal diameter $d_{\text {min }}$ of water drop), and 0 , if output particle is water drop.

Thus, $C_{\text {out }}$ is defined as

$$
\frac{\rho \sum_{k=1}^{N(\Delta t)} H_{d_{\min }}\left(d_{\mathrm{k}}\right) \frac{\pi d_{\mathrm{k}}^{3}}{6}}{\Delta t} .
$$

Criterion of passing of particle is radius vector of particles belonging to a set of points of the section of the section of the output pipe:

$$
\mathbf{r}_{\mathrm{p}}(t) \in S, \quad \mathbf{r}_{\mathrm{p}}(t)=\int_{0}^{\infty} \mathbf{v}_{\mathrm{p}}(t) \partial t
$$

where $t$ is time, $\mathrm{s} ; \mathbf{r}_{\mathrm{p}}(t)$ is vector of point of particle, $\mathrm{m}$; $\mathbf{v}_{\mathrm{p}}(t)$ is vector of particle velocity, $\mathrm{m} / \mathrm{s} ; S$ is the surface of output pipe cross-section.

From the obtained nominal diameters and number of particles passing through the section of the output pipe and expression (8) the dust extraction plant efficiency is defined as:

$$
n=\left(1-\frac{\rho \sum_{k=1}^{N(\Delta t)} H_{d_{\min }}\left(d_{\mathrm{k}}\right) \frac{\pi d_{\mathrm{k}}^{3}}{6}}{\alpha G \Delta t}\right) \cdot 100 \%
$$

As presented model allows to estimate efficiency of gas cleaning plant depending on its geometric sizes and parameters of powder-gas flow, further we present the analysis of efficiency of the typical dust extraction 
plant on the base of Venturi tube, which is schematically shown in Fig. 2 and having performance features presented in Table 1.

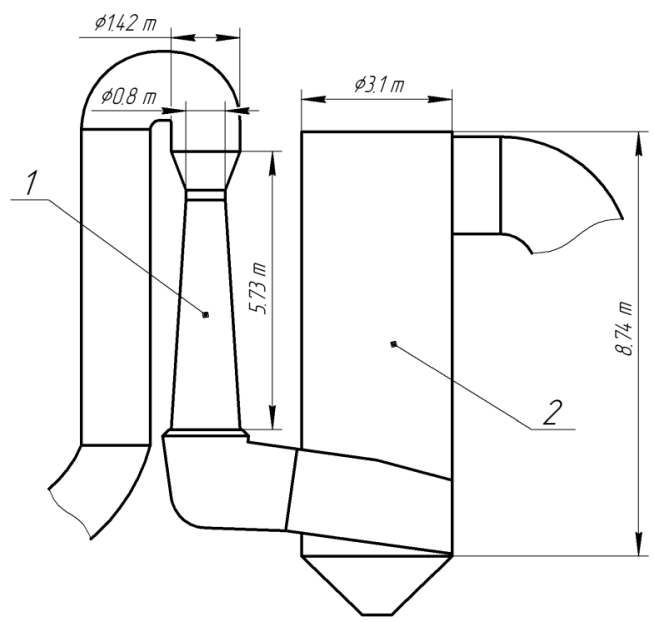

Fig. 2. Scheme of model dust extraction plant, 1 - Venturi tube; 2 - drop catcher.

Table 1. Performance features of the model dust extraction plant.

\begin{tabular}{|l|c|}
\hline $\begin{array}{l}\text { Gas consumption }\left[\mathrm{Nm}^{3} / \mathrm{h}\right] \\
\left(\mathrm{m}^{3} / \mathrm{h} \text { at normal conditions }\right)\end{array}$ & $400-500$ \\
\hline Density of gas at the input $\left[\mathrm{kg} / \mathrm{m}^{3}\right]$ & 0.78 \\
\hline Gas temperature at the input $\left[{ }^{\circ} \mathrm{C}\right]$ & 168 \\
\hline Gas temperature at the output $\left[{ }^{\circ} \mathrm{C}\right]$ & $80-90$ \\
\hline Water consumption for spraying $[\mathrm{t} / \mathrm{h}]$ & 10 \\
\hline Opening angle of water sprayer $\left[{ }^{\circ}\right]$ & 120 \\
\hline Mean diameter of spraying drops $d_{10}[\mu \mathrm{m}]$ & 200 \\
\hline $\begin{array}{l}\text { Residual dust content at the output of the } \\
\text { dust extraction plant }(\text { if the dust content } \\
\text { at the input of the dust extraction plant is } \\
\left.17 \mathrm{~g} / \mathrm{Nm}^{3}\right)\left[\mathrm{g} / \mathrm{Nm}^{3} \text { ] }\right.\end{array}$ & $0.6-0.87$ \\
\hline
\end{tabular}

Graphical interpretation of the calculation results of motion of solid particles and water particles obtained by solving Eq. (5) in the typical dust extraction plant is shown in Figs. 3-5.

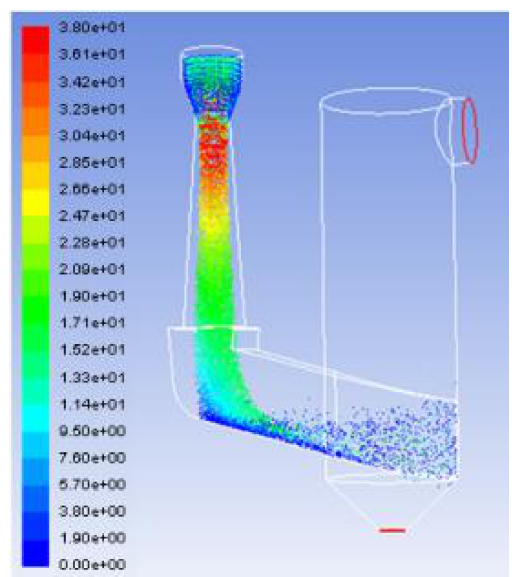

Fig. 3. Velocity distribution of water drops (in $\mathrm{m} / \mathrm{s}$ ). a)

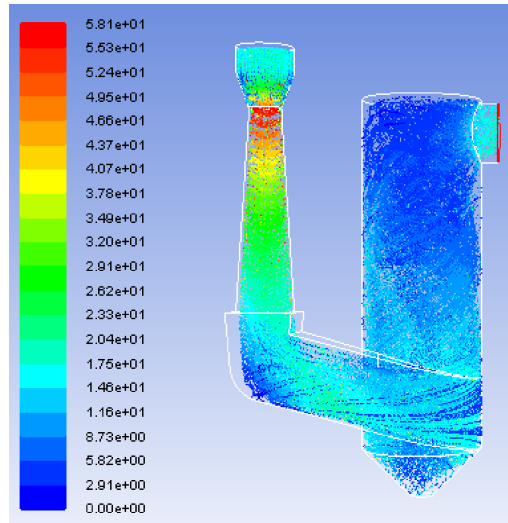

b)

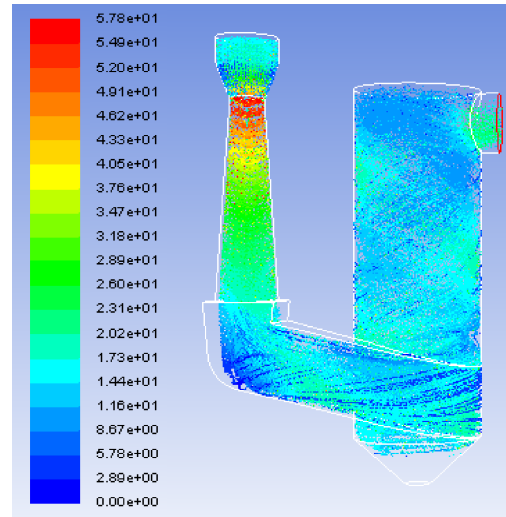

c)

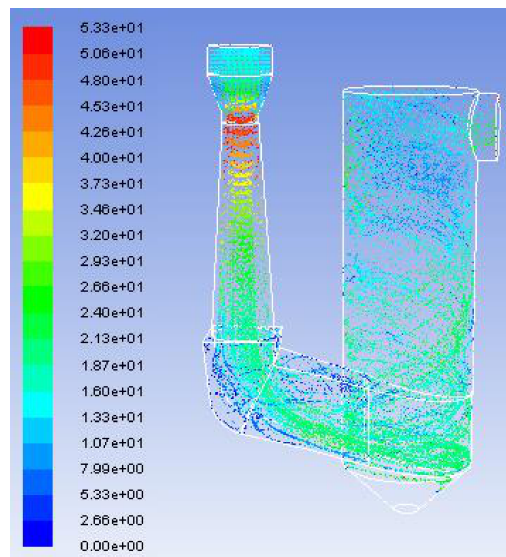

d)

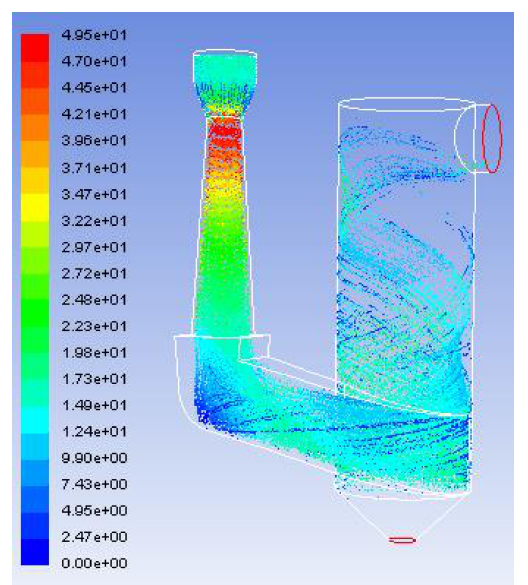

Fig. 4. Velocity distribution of ash particles of different conventional diameters (in $\mathrm{m} / \mathrm{s}$ ): a) $2 \mu \mathrm{m}$, b) $10 \mu \mathrm{m}$, c) $40 \mu \mathrm{m}$, d) $90 \mu \mathrm{m}$. 
a)

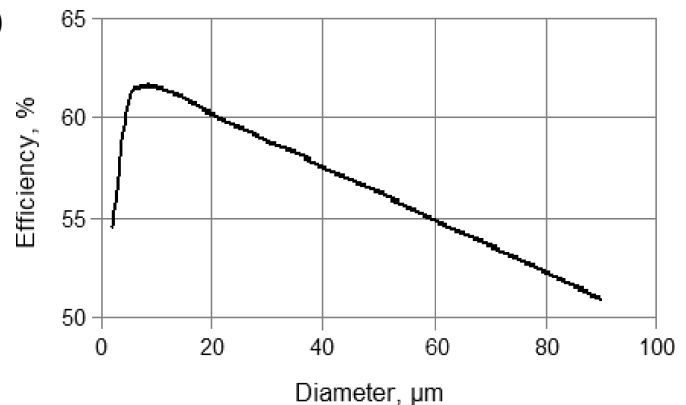

b)

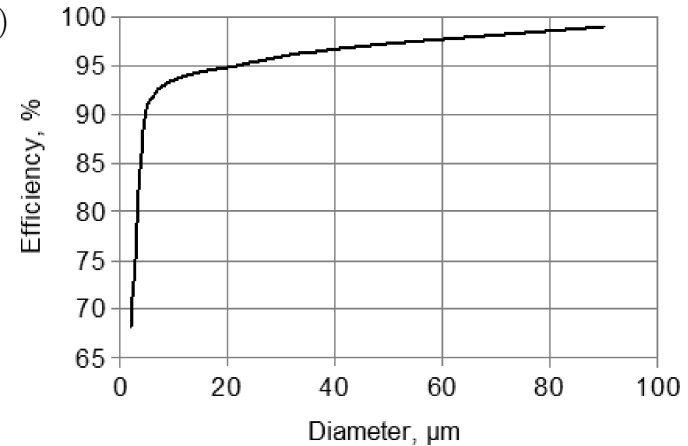

Fig. 5. Dependence of efficiency of Venturi tube (a) and all dust extraction plant (b).

As it follows from Fig. 3, water drops with the diameter of more than $150 \mu \mathrm{m}$ with ash particles are caught in the lower part of the drop catcher. At that the main deposition of ash particles on water drops occurs in the neck of Venturi tube, where the most difference (up to $20 \mathrm{~m} / \mathrm{s}$ ) of ash particle velocities (Fig. 4) and water drops (Fig. 3) is achieved.

Figure 5 shows the dependences of efficiency of Venturi tube and dust extraction plant in a whole on the conventional diameter of ash particles.

As it follows from presented dependences the efficiency of ash particle absorption in Venturi tube (1st stage of cleaning) does not exceed $65 \%$. It is caused by limitation of difference of ash and water velocities (no more than $20 \mathrm{~m} / \mathrm{s}$ ) and area of efficient section of particle collision. However, Fig. 5b shows that efficiency of all dust extraction plant (Venturi tube + drop catcher) exceeds $95 \%$ for the particles with the size of more than $20 \mu \mathrm{m}$ due to their high inertia leading to deposition of such particles on the walls of the drop catcher.

Carried out analysis allows to state that efficiency of particle collection with the size of less than $5 \mu \mathrm{m}$ in the dust extraction plant does not exceed $75 \%$ and the reason of it is insufficient deposition degree of ash particles on water drops in Venturi tube (no more than $65 \%)$.

Increase of deposition degree should be provided by additional interaction of ash and water particles under the action of ultrasonic vibrations. Further studies are aimed at the analysis of dust collection at the presence of ultrasonic action for determination of introduction conditions and modes of action.

\section{Efficiency increase of particle collection under action of ultrasound}

The analysis of dust collection efficiency at the realization of ultrasonic influence is performed by the same approach as well as without ultrasonic influence (see Sec. 2). However, analysis of efficiency at the ultrasonic influence unlike previous analysis takes into account additional forces caused by both periodical disturbance of gas flow velocity and secondary reflection of ultrasonic vibrations from neighboring particles. To take into account additional forces the equation of particle motion (5) is supplemented by the third summand in the right part $\left(\mathbf{F}_{e}\right)$ characterizing these forces (9) according to the second Newton law.

$$
\begin{aligned}
m_{\mathrm{p}} \frac{\partial \mathbf{v}_{\mathrm{p}}}{\partial t}= & 3 \pi \mu d C_{\mathrm{cor}}\left(\mathbf{v}_{\mathrm{f}}-\mathbf{v}_{\mathrm{p}}\right) \\
& +\frac{\pi d^{3} \rho_{\mathrm{f}}}{12}\left(\frac{\partial \mathbf{v}_{\mathrm{f}}}{\partial t}-\frac{\partial \mathbf{v}_{\mathrm{p}}}{\partial t}\right)+\mathbf{F}_{e}(\mathbf{r}),
\end{aligned}
$$

where $m_{p}$ is the particle mass, $\mathrm{kg} ; d$ is the particle diameter, $\mathrm{m} ; v$ is the velocity, $\mathrm{m} ; \mu$ is the dynamic substance viscosity of the basic phase, $\mathrm{Pa} \cdot \mathrm{s} ; C_{\text {cor }}$ is the coefficient of viscous resistance; $\mathbf{r}$ is radius-vector of particle, $\mathrm{m}$.

Many papers (VINCENT, 1987; KUdRYASHOVA et al. 2013; DANSER, Neumann, 1949; KhMELEV et al., 2008; ZHANG et al., 2012) are devoted to the calculation of additional force $\mathbf{F}_{e}(\mathbf{r})$ acting upon the particle at the application of ultrasonic vibrations and presence of neighboring particles, they analyze the interaction of the particles different in size and shape. As the shape of harmful emission particles differs from spherical and it is closed to elongated one, the particle is assumed to be ellipsoid of revolution.

Additional force acting upon ellipsoidal particle according to Oseen is defined as (CHERNOv, 2004):

$$
\begin{aligned}
\mathbf{F}_{\mathrm{e}}= & \frac{4 \pi d \mu\left(W^{2}-1\right)^{3 / 2}}{W} \\
& \times\left(\frac{\cos ^{2} \theta}{\left(W^{2}-2\right) \arctan \sqrt{W^{2}-1}-\sqrt{W^{2}-1}}\right. \\
& \left.+\frac{\sin ^{2} \theta}{\left(3 W^{2}-2\right) \arctan \sqrt{W^{2}-1}-\sqrt{W^{2}-1}}\right) \\
& \times \mathbf{U}(\mathbf{r}, t),
\end{aligned}
$$

where $d$ is the largest diameter of ellipsoidal particle, $\mathrm{m} ; \mu$ is the viscosity of gas medium, $\mathrm{Pa} \cdot \mathrm{s} ; \theta$ is the angle between the smallest particle semi-axis and direction of ultrasonic field, rad; $W$ is the ratio of the larger semi-axis length of the particle to the smaller one; $\mathbf{U}$ is the disturbance of gas flow velocity.

For the calculation of value of disturbance of gas flow velocity being a part of the expression for force $\mathbf{F}_{e}$ two dominating mechanisms of particle interaction 
under the action of ultrasonic vibrations: orthokinetic (caused by difference between oscillation amplitudes of particles of different sizes) and hydrodynamic (caused by radiation pressure from wave reflected from neighbour particle) under the action of Oseen force were taken into consideration (CHERNOV, 2004).

According to the mechanisms disturbance of velocity is defined by the expression (11) which is followed from the fact that the disturbances like harmonic oscillations:

$$
\mathbf{U}(\mathbf{r}, t)=\left(\mathbf{U}_{1}(\mathbf{r})+\mathbf{U}_{2}(\mathbf{r})\right) \cos (\omega t+\varphi(\mathbf{r})),
$$

where $\mathbf{U}_{1}$ is the amplitude of disturbance of gas flow velocity at the side of primary ultrasonic field, $\mathrm{m} / \mathrm{s}$; $\mathbf{U}_{2}$ is the amplitude of disturbance of gas flow velocity generated at the reflection of ultrasonic waves from the surface of neighboring particles, $\mathrm{m} / \mathrm{s} ; t$ is the time, $\mathrm{s}$; $\varphi$ is the phase shift of vibrational velocity depending on the point of the internal volume of Venturi tube.

The phase shift of gas flow vibrational velocity $\varphi$ is defined on the base of the following expression:

$$
\varphi(\mathbf{r})=\operatorname{Arg}\left(\frac{\nabla P(\mathbf{r})}{i \omega \rho}\right),
$$

where $\rho$ is the density of air, $\mathrm{kg} / \mathrm{m}^{3} ; P$ is the complex amplitude of sound pressure in liquid medium, $\mathrm{Pa}$.

The amplitude of disturbance of gas flow velocity $\mathbf{U}_{1}(\mathbf{r})$ caused by ultrasonic action is defined as:

$$
\mathbf{U}_{1}(\mathbf{r})=\operatorname{Re}\left(\frac{\nabla P(\mathbf{r})}{i \omega \rho}\right) .
$$

The amplitude of disturbance of velocity generated at the reflection of ultrasonic waves from the surface of neighbouring particles is defined as (CHERNOv, 2004):

$$
\begin{aligned}
\mathbf{U}_{2}(\mathbf{r})= & \sum_{k=1}^{N}\left[\frac{3 \nu R_{\mathrm{k}} V_{\mathrm{k}}\left(\mathbf{r}_{\mathrm{k}}-\mathbf{r}\right)}{2\left|V_{\mathrm{k}}\right|\left|\mathbf{r}_{\mathrm{k}}-\mathbf{r}\right|^{3}}\right. \\
& \cdot\left[1-e^{-\frac{\left|\mathbf{r}_{\mathrm{k}}-\mathbf{r}\right|}{2 \nu}\left(\left|V_{\mathrm{k}}\right|-V_{\mathrm{k}}\left|\frac{\left(\mathbf{k}, \mathbf{r}_{\mathrm{k}}-\mathbf{r}\right)}{k\left|\mathbf{r}_{\mathrm{k}}-\mathbf{r}\right|}\right|\right)}\right. \\
& \left.\cdot\left[1+\frac{\left|\mathbf{r}_{\mathrm{k}}-\mathbf{r}\right|}{2 \nu}\left(\left|V_{\mathrm{k}}\right|+V_{\mathrm{k}}\left|\frac{\left(\mathbf{k}, \mathbf{r}_{\mathrm{k}}-\mathbf{r}\right)}{k\left|\mathbf{r}_{\mathrm{k}}-\mathbf{r}\right|}\right|\right)\right]\right] \\
& -\frac{3 R_{\mathrm{k}} V_{\mathrm{k}}^{2}}{4\left|\mathbf{r}_{\mathrm{k}}-\mathbf{r}\right|\left|V_{\mathrm{k}}\right|} \sqrt{1-\left|\frac{\left(\mathbf{k}, \mathbf{r}_{\mathrm{k}}-\mathbf{r}\right)}{k\left|\mathbf{r}_{\mathrm{k}}-\mathbf{r}\right|}\right|^{2}} \\
& \cdot \mathrm{e}^{\left.-\frac{\left|\mathbf{r}_{\mathrm{k}}-\mathbf{r}\right|}{2 \nu}\left(\left|V_{\mathrm{k}}\right|-V_{\mathrm{k}}\left|\frac{\left(\mathbf{k}, \mathbf{r}_{\mathrm{k}}-\mathbf{r}\right)}{k\left|\mathbf{r}_{\mathrm{k}}-\mathbf{r}\right|}\right|\right) \mathbf{e}_{\mathrm{k} \theta}\right]} \\
& \cdot\left(1+\frac{3 R_{\mathrm{k}}\left|V_{\mathrm{k}}\right|}{8 \nu}\right),
\end{aligned}
$$

where $\nu$ is the kinematic viscosity of gas medium, $\mathrm{m}^{2} / \mathrm{s}$; $R_{\mathrm{k}}$ is the radius $k$ of the neighbouring particle, $\mathrm{m} ; V_{\mathrm{k}}$ is the projection of gas flow velocity to the wave vector of the acoustic field in the location of $k$ particle, $\mathrm{m} / \mathrm{s}$; $\mathbf{r}$ is the position of center of studied particle, $\mathrm{m} ; \mathbf{r}_{k}$ is the position of center $k$ of neighbouring particle, m; $\mathbf{k}$ is the wave vector of the ultrasonic field, $\mathrm{m}^{-1} ; \mathbf{e}_{\mathrm{k} \theta}$ is the unit vector, which is perpendicular to the vector $\mathbf{r}_{\mathrm{k}}-\mathbf{r}$.

Being a part of the expression for the phase shift (12) and amplitude (13) of vibrational velocity of gas flow the complex amplitude of sound pressure $P(\mathbf{r})$ is defined on the base of wave equation (15) with boundary conditions on the walls of gas cleaning plant (16) and the surface of the ultrasonic radiator (17).

$$
\begin{aligned}
\Delta P+k_{*}^{2} P & =0, \\
0 & =\left(\nabla p, \mathbf{n}_{2}\right), \\
\omega^{2} \rho A_{\mathrm{n}}(\mathbf{r}) & =\left(\nabla p, \mathbf{n}_{1}\right),
\end{aligned}
$$

where $k_{*}$ is the efficient wave number of gas medium taking into account absorption of ultrasonic vibrations in medium, $\mathrm{m}^{-1} ; p$ is the complex amplitude of sound pressure in gas medium, $\mathrm{Pa} ; \mathbf{n}_{1}$ is the normal vector to the wall of Venturi tube; $\mathbf{n}_{2}$ is the normal vector to the radiator surface; $f$ is the frequency of ultrasonic vibrations; $\rho$ is the density of gas medium, $\mathrm{kg} / \mathrm{m}^{3} ; A_{\mathrm{n}}(\mathbf{r})$ is the function of distribution of normal vibrations on the surface of the radiator, $\mathrm{m}$.

The Eqs. (15)-(17) are solved by boundary element method. Obtained data allows to determine mode of ultrasonic action, conditions of introduction of ultrasonic vibrations and number of the radiators, which is necessary for providing required action zone, i.e. for required efficiency of gas cleaning plant on the base of Venturi tube with the ultrasonic radiators.

\section{Determination of dependences of coagulation efficiency on the modes of ultrasonic action}

The efficiency of particles aggregation under the action of ultrasonic vibrations is determined by frequency of ultrasonic influence and level of sound pressure.

Figure 6 shows the dependence of efficiency of Venturi tube at different frequencies of ultrasonic action for collection of particles of various sizes (if the particles have monodispersed composition) at the level of

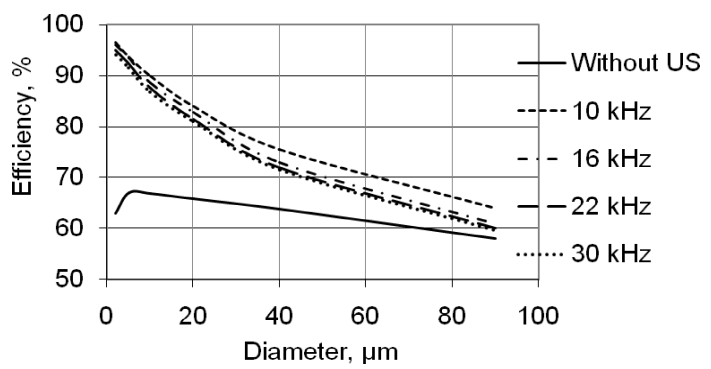

Fig. 6. Dependence of Venturi tube efficiency on the size of particles at different frequencies. 
sound pressure of $145 \mathrm{~dB}$. At these and following calculations the parameters of gaseous phase are presented in Table 1, and the total dust content at dust extraction plant input independently on particle dispersed compostition is $17 \mathrm{~g} / \mathrm{Nm}^{3}\left(17 \mathrm{~g} / \mathrm{m}^{3}\right.$ at normal conditions).

As the realization of ultrasonic action at the frequencies of lower than $22 \mathrm{kHz}$ can be dangerous for the personnel and radiation at higher frequency is characterized by increased damping in the medium of solid particles and water drops, further we present the dependences of coagulation efficiency at the collection of monodispersed ash particles with the size of $2 \mu \mathrm{m}$ on the level of sound pressure at the frequency of vibrations of $22 \mathrm{kHz}$ (Fig. 7).

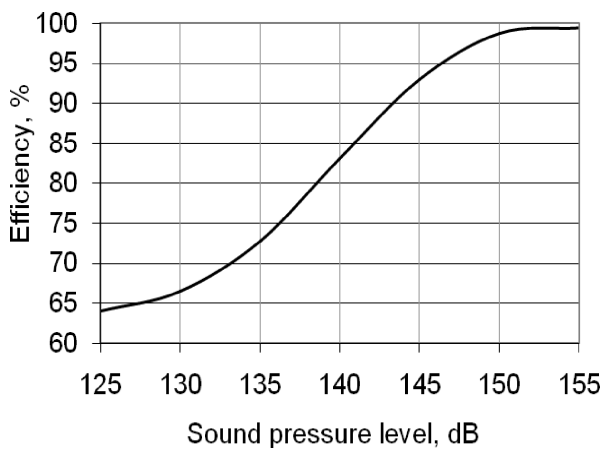

Fig. 7. Dependence of Venturi tube efficiency on the level of sound pressure (the size of ash particles is $2 \mu \mathrm{m})$.

From presented dependences it follows that to provide essential efficiency increase of deposition of finedispersed particles on water drops (for instance, from $65 \%$ to $75 \%$ ) it is necessary to realize the action of ultrasonic vibrations at the level of sound pressure of no less than $135 \mathrm{~dB}$. At the same time the growth of level of sound pressure to more than $155 \mathrm{~dB}$ does not lead to the efficiency increase of Venturi tube due the rise of probability of secondary dispersion of the aggregates.

Figure 8 shows the dependences of Venturi tube efficiency (Fig. 8a) and residual dust content of gas flow (Fig. 8b) on the size of monodispersed particles at different levels of ultrasonic action. Figure 9 presents similar dependences of efficiency of all gas cleaning plant.

The action of ultrasonic vibrations with the level of sound pressure of $150 \mathrm{~dB}$ provides double reduction of dust content at the output of Venturi tube at the collection of particles of up to $20 \mu \mathrm{m}$ and in 1.5 times at the collection of particles of more than $20 \mu \mathrm{m}$. At that the most effect of gas cleaning can be achieved at the collection of particles of less than $5 \mu \mathrm{m}$. The efficiency of particles collection of $2 \mu \mathrm{m}$ at the action with the level of sound pressure of $150 \mathrm{~dB}$ increased up to $99 \%$ that is considerably higher than efficiency of cleaning without ultrasonic action (no more than $75 \%$ ). a) efficiency of ash particle collection by Venturi tube

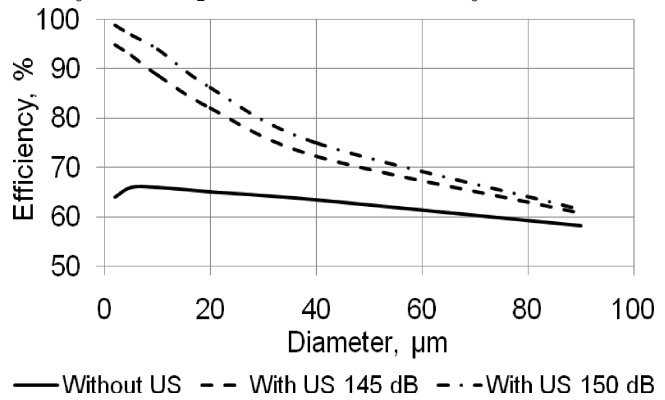

b) dust content of gas flow at the output of Venturi tube

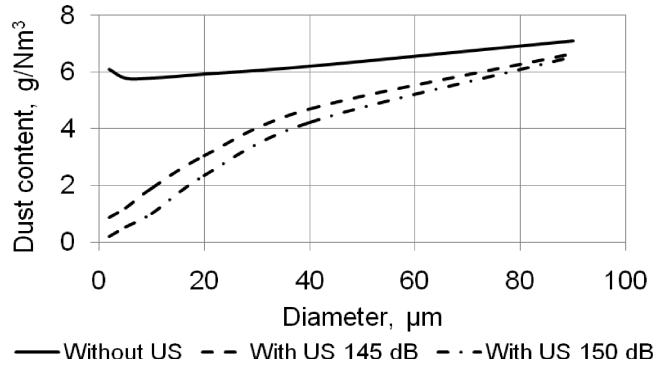

Fig. 8. Dependences of efficiency (a) and dust content (b) on the size of ash particles.

a) efficiency of the gas cleaning plant

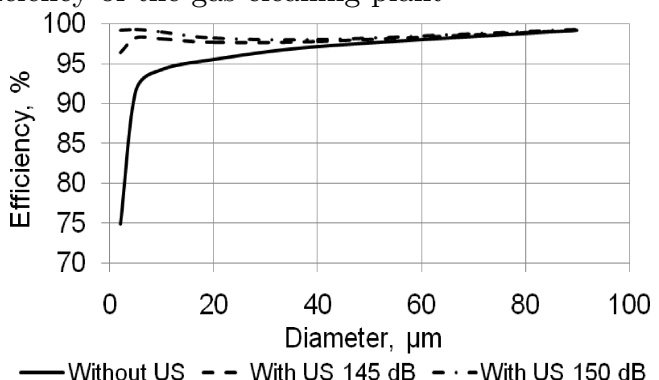

b) dust content of gas flow at the output

of the drop catcher

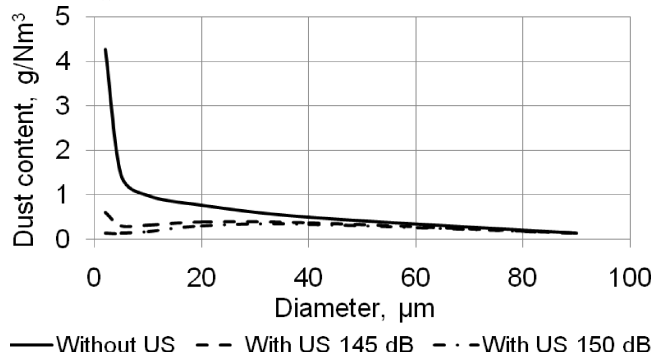

Fig. 9. Dependences of efficiency and dust content at the output of the gas cleaning plant on the size of ash particles at different levels of sound pressure.

Ultrasonic action leads to decrease of residual mass of fine-dispersed $(2-5 \mu \mathrm{m})$ particles, which do not combine with water drops, in 6 times at the level of sound pressure of $150 \mathrm{~dB}$ and in 4 times at the level of sound pressure of $145 \mathrm{~dB}$.

As typical Venturi tube is rather tall (more than $10 \mathrm{~m}$ ) at the diameter of $1.5 \mathrm{~m}$, the application of the 
radiators requires to choose direction of ultrasonic vibrations introduction in order to provide maximum uniformity of the field and determine the area of radiation surface for the coagulation zone of maximum size.The results of calculations of gas cleaning efficiency depending on direction of ultrasonic vibrations introduction are presented below.

\section{Determination of dependences of efficiency on direction of ultrasonic vibrations entry}

To provide even distribution of energy of ultrasonic action with previously determined level of sound pressure it is necessary to locate the radiators in a proper way. The problem of choice of ultrasonic vibrations directions was solved at the example of two radiators (for symmetry of the picture) in the form of disk generating vibrations with maximum level of sound pressure of $150 \mathrm{~dB}$ at the frequency of $22 \mathrm{kHz}$ installed at different angles to the axis of Venturi tube. The radiators are placed in the cap of Venturi tube (Fig. 10) where maximum concentration of ash particles and water drops is provided.

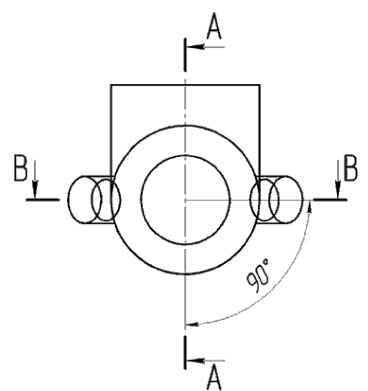

Fig. 10. Scheme of installation of two radiators into Venturi tube (top view).

Obtained distributions of sound pressure in mutually perpendicular section planes (A-A and B-B) depending on the angle of radiator installation determining direction of radiation of ultrasonic vibrations are shown in Fig. 11. The distributions were obtained by Eqs. (15)-(17) and visualized by colour legend of sound pressure level. From presented distributions it is evident that the uniformity of sound field in Venturi tube at maximum level of sound pressure is provided at the entry of vibrations at the angle of 45 degrees to the axis of the tube.

Figures 12 and 13 show dependences of mean value and mean-square deviation of sound pressurein all volume of the tube. As it follows from the dependences, at the entry angle of 45 degrees maximum of mean level of sound pressure and minimum mean-square deviation are achieved.

Calculations of Venturi tube efficiency at the collection of monodisperse ash at the different angles of installation of ultrasonic radiators prove the correctness a) 30 degrees

b) 45 degrees
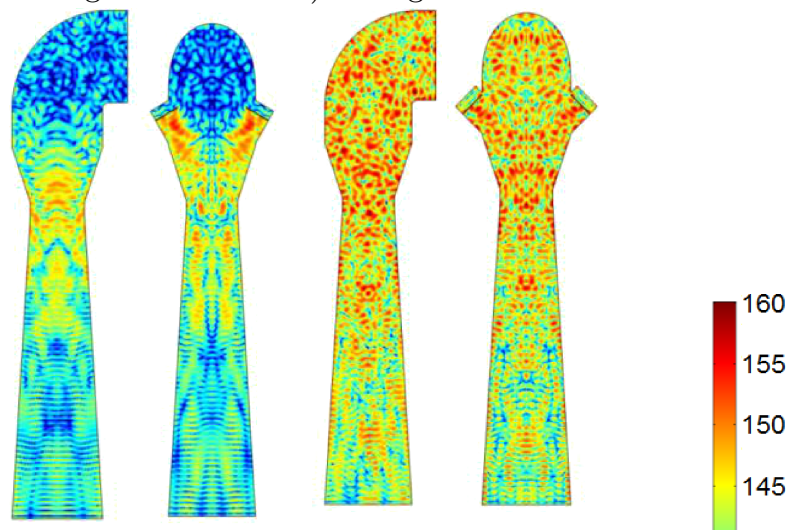

A-A

B-B

A-A

B-B

d) 90 degrees
A-A

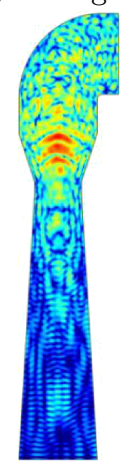

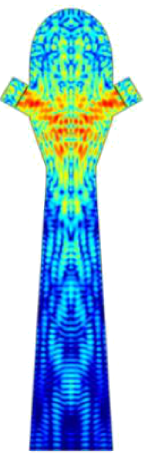

B-B

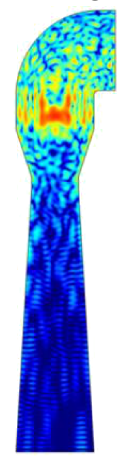

A-A

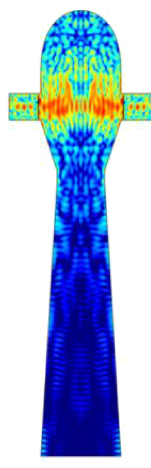

B-B
Fig. 11. Distribution of sound pressure at different angles of ultrasonic vibrations entry.

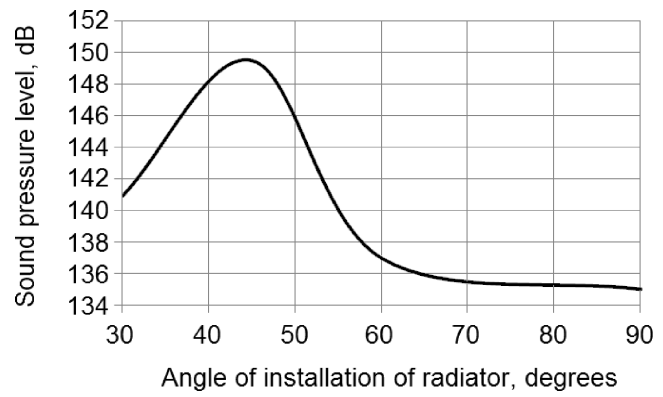

Fig. 12. Dependence of mean value of sound pressure level on entry angle of ultrasonic vibrations.

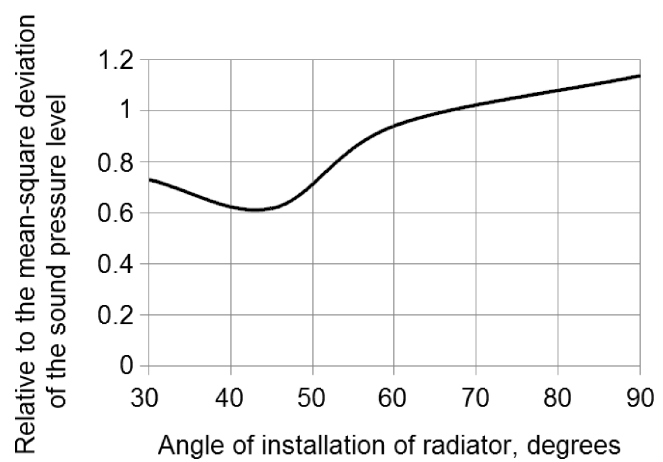

Fig. 13. Dependence of mean-square deviation of sound pressure level on entry angle of ultrasonic vibrations. 
of choice of entry angle of ultrasonic vibrations. Dependences of efficiency of Venturi tube and gas cleaning plant in a whole on the conventional diameter of ash particles at different entry angles of ultrasonic vibrations are presented in Figs. 13a, 14a, respectively. Figures $13 \mathrm{~b}$ and $14 \mathrm{~b}$ show the dependences of residual dust content at the output of Venturi tube and all dust

a)

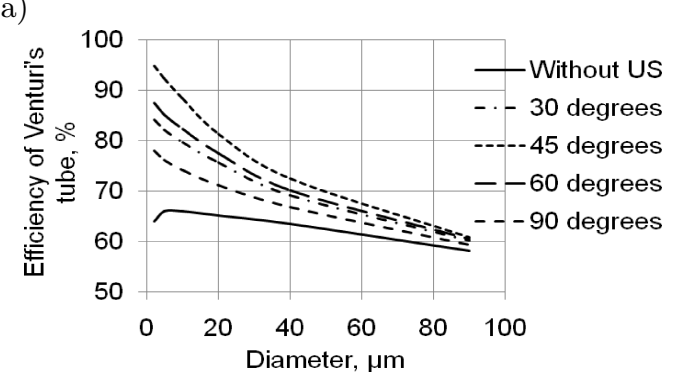

b)

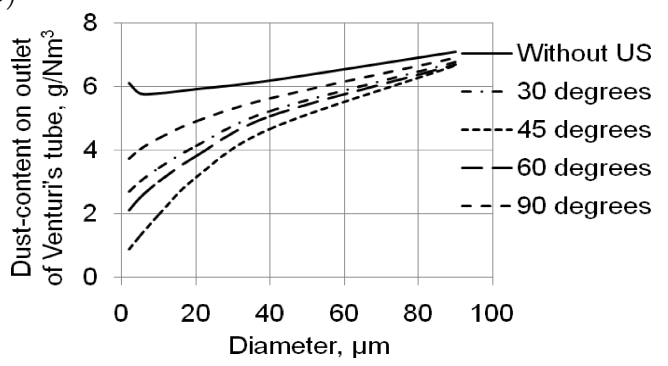

Fig. 14. Dependence of Venturi tube efficiency (a) and dust content (b) at the output of the tube on particles size at different entry angles of ultrasonic vibrations.

a)

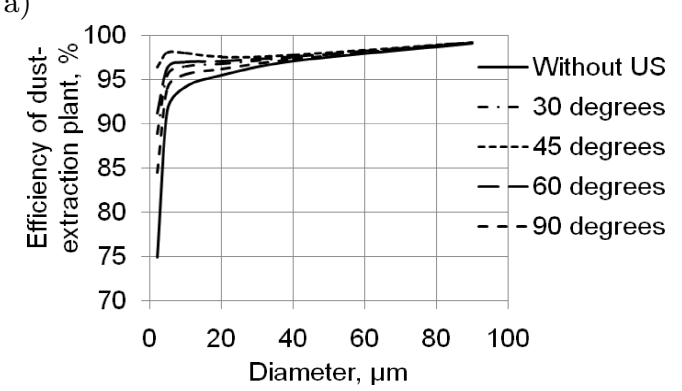

b)

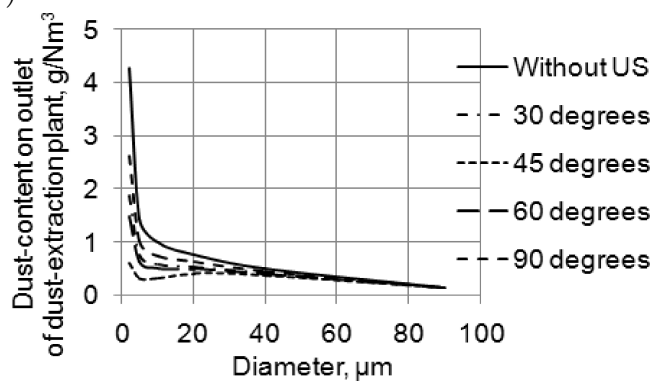

Fig. 15. Dependence of dust extraction plant efficiency (a) and dust content at its output (b) on particles size at different entry angles of vibrations (monodisperse ash with initial dust content of $\left.17 ; \mathrm{g} / \mathrm{Nm}^{3}\right)$. extraction plant, i.e. masses of ash particles, which do not coagulate with drops of spraying water.

From obtained results it follows, that at the entry of ultrasonic vibrations at 45 degrees to the axis of the tube, efficiency of the dust extraction plant achieves $98 \%$ for the particles of $2 \mu \mathrm{m}$.

Further we determine conditions of providing the maximum size of the action zone, which is characterized by energy of entered vibrations (i.e. size of the radiating surface and the number of the radiators).

\section{Determination of dependences of dust extraction plant efficiency on number and size of the radiators}

To determine the efficiency of cleaning at the application of several sources of ultrasonic vibrations $(2,4$ or 6 pieces, see Fig. 16) with the radiating surface area in the range of $0.08 \ldots 0.14 \mathrm{~m}^{2}$ (the diameters of the radiators from 320 to $418 \mathrm{~mm}$ ) operating at the frequency of $22 \mathrm{kHz}$ the calculations of distribution of sound pressure were carried out.

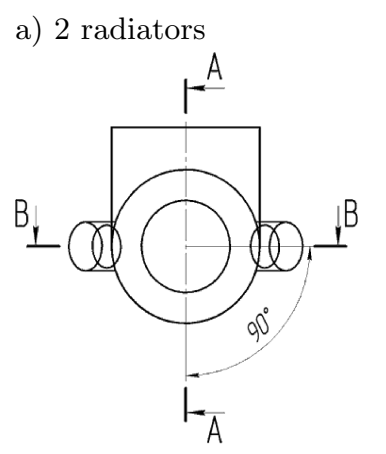

b) 4 radiators

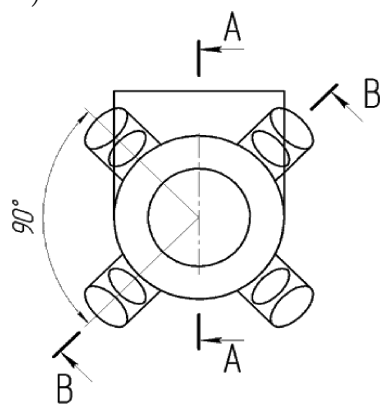

c) 6 radiators

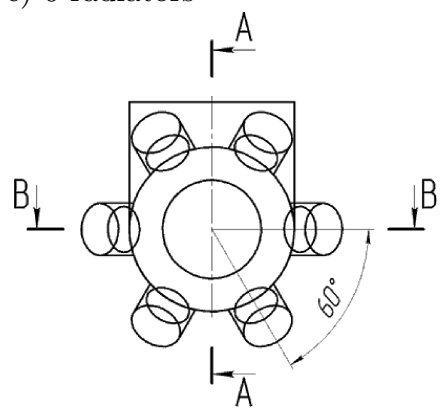

Fig. 16. Scheme of installation of various number of radiators into Venturi tube (top view).

Figure 17 shows distributions at the application of two sources of ultrasonic vibrations with different radiating surface. The estimation of the dust extraction plant efficiency at the application of two sources of ultrasonic action with different area is shown in Fig. 18.

As it follows from the presented dependences, ultrasonic action allows to provide dust extraction plant efficiency of more than $95 \%$ in the range of the conventional diameters of the particles of $2 \ldots 90 \mu \mathrm{m}$. At that 
a) $0.08 \mathrm{~m}^{2}$

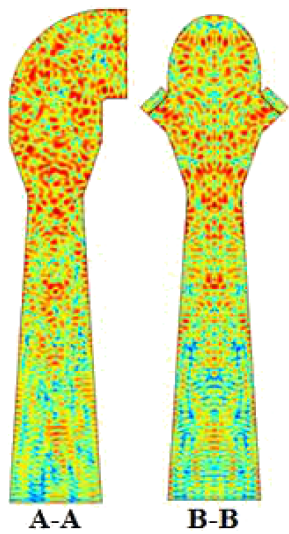

b) $0.11 \mathrm{~m}^{2}$

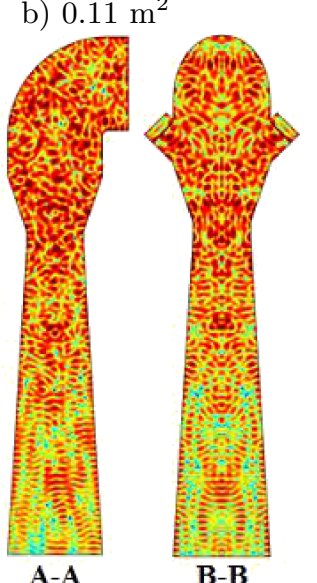

c) $0.14 \mathrm{~m}^{2}$

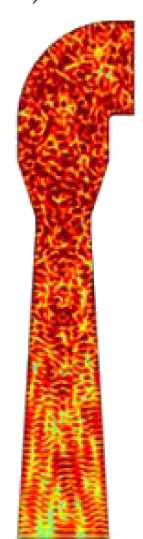

$\mathbf{A}-\mathbf{A}$

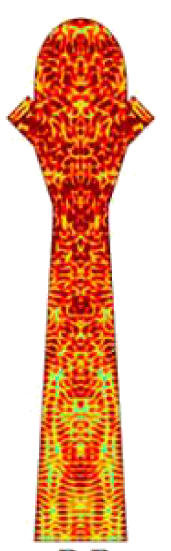

B-B

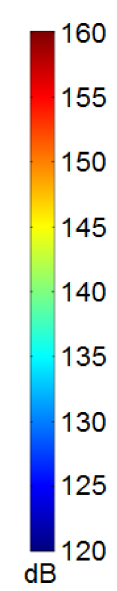

Fig. 17. Distribution of sound pressure at two sources of ultrasonic action with different radiating surface area.

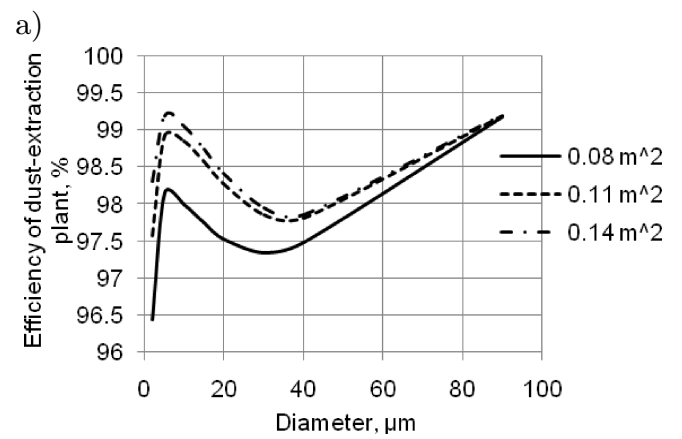

b)

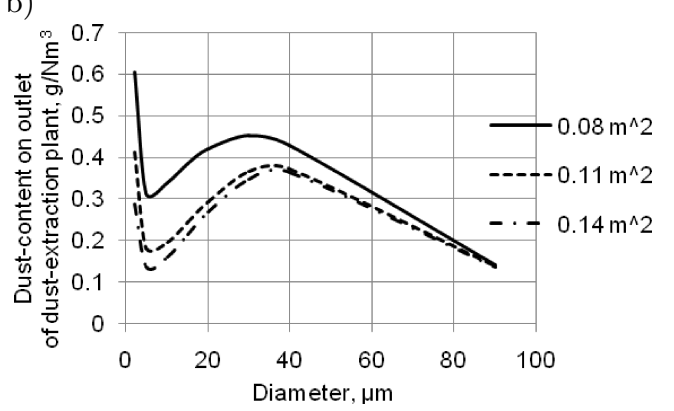

Fig. 18. Dependence of the dust extraction plant efficiency (a) and dust content at its output (b) on size of ash particles at two sources of ultrasonic action and different radiating surface areas. with the increase of particles size efficiency asymptotically approaches to $100 \%$ due to the growth of inertia providing their collection by the drop catcher. However, in the range of sizes $2 . .50$ presented graphs have one local maximum and one local minimum.

Regardless of radiating surface area local efficiency maximum (dust content minimum) is present at the particle size of $5 \mu \mathrm{m}$. It is caused by the fact that at smaller particle sizes the efficiency of the drop catcher drops, at larger particle sizes the degree of particle involvement into vibrational motion decreases and, respectively, the probability of their collision with water drops in Venturi tube reduces. At that drop catcher efficiency does not exceed $95 \%$ up to the particle size of $40 \mu \mathrm{m}$. Beginning with the size of $40 \mu \mathrm{m}$ further efficiency fall of collision of ash particles with water drops (see Figs. 14, 15) is observed, however efficiency of dust extraction plant in a whole achieves local minimum. This minimum is due to the fact that low efficiency of Venturi tube is leveled by high efficiency of the drop catcher for large particles (40 $\mathrm{m}$ and more).

As initial ash powder supplied to the input of the dust extraction plant is determined by the type of burnt coal, further Figs. 19, 20 show ash powder obtained at the burning of coal from Kharanor coal deposit and Ekibastuz field (SkrYABINA, 1980). The powder at the output of the dust extraction plant at the installation of two sources of ultrasonic vibrations

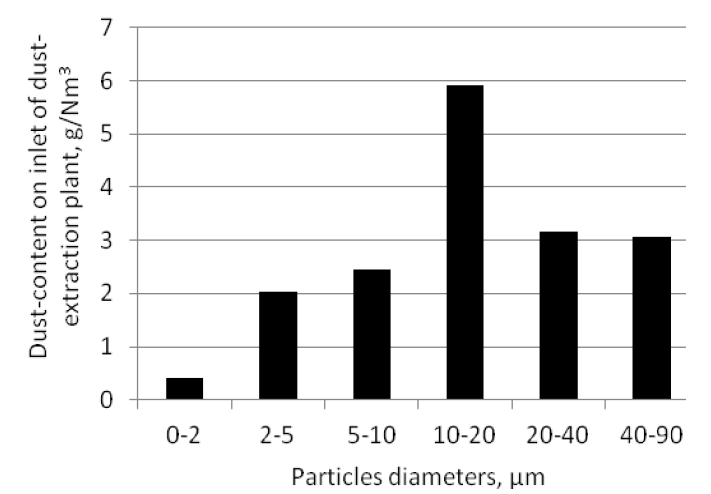

Fig. 19. Initial ash powder formed at burning of coal from Kharanor coal deposit.

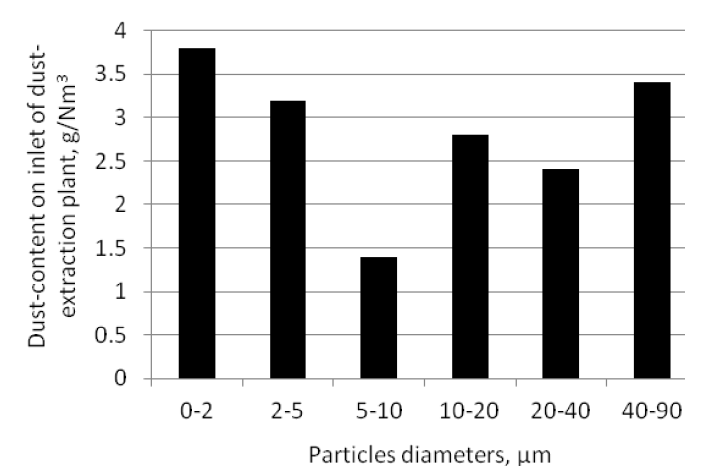

Fig. 20. Initial ash powder formed at burning of coal from Ekibastuz field. 
of various areas is presented in Fig. 21. As it follows from the presented results, total residual dust content at the output of the dust extraction plant does not exceed allowable value $\left(0.3 \mathrm{~g} / \mathrm{Nm}^{3}\right)$ at the radiating surface area of no less than $0.14 \mathrm{~m}^{2}$ in of ultrasonic vibrations.

$$
\text { a) }
$$

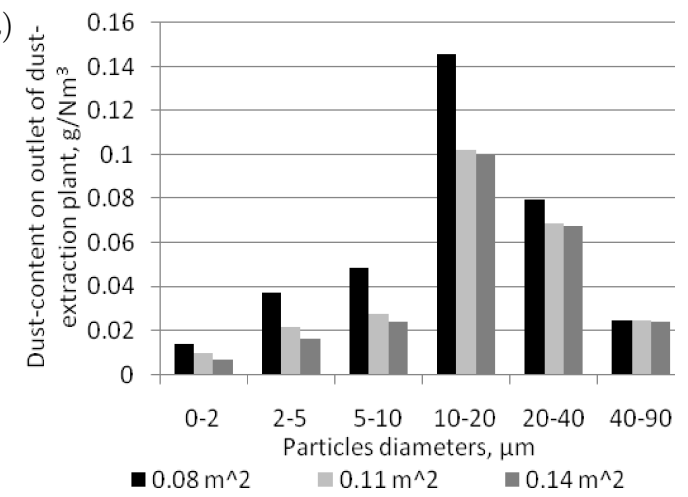

b)

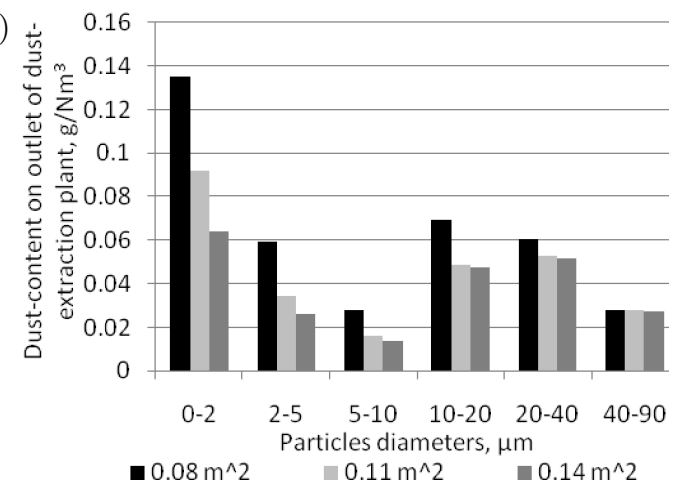

Fig. 21. Fraction ash composition at the output of the dust extraction plant at the application of two sources of ultrasonic action of various areas at ash collection formed at burning of coal from Kharanor coal deposit (a) and Ekibastuz field (b).

Distribution of sound pressure at the application of four radiators of ultrasonic vibrations of various areas is shown in Fig. 22.

Dependences of dust extraction plant efficiency and dust content at the output in the case of application of four radiators of various areas are presented in Figs. 23, 24.

According to the presented results fulfillment of requirements of ecological standards is achieved at the use of four radiators of ultrasonic vibrations with the radiating surface area of $0.11 \mathrm{~m}^{2}$.

The application of six sources of ultrasonic action provides distribution shown in Fig. 25.

At that sound pressure level of $145 \mathrm{~dB}$ is not achieved in the lower part of the diffuser of Venturi tube even at the installation of six radiators of ultrasonic vibrations of $0.08 \mathrm{~m}^{2}$. At the application of six radiators of ultrasonic vibrations with the area of $0.14 \mathrm{~m}^{2}$ sound pressure level exceeds $155 \mathrm{~dB}$ even in the confusor that is why further increase of radiators number of ultrasonic vibrations does not make sense. a) $0.08 \mathrm{~m}^{2}$

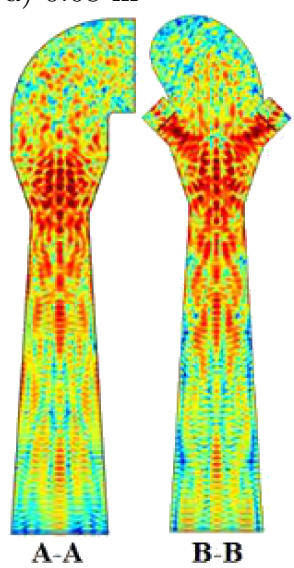

b) $0.11 \mathrm{~m}^{2}$

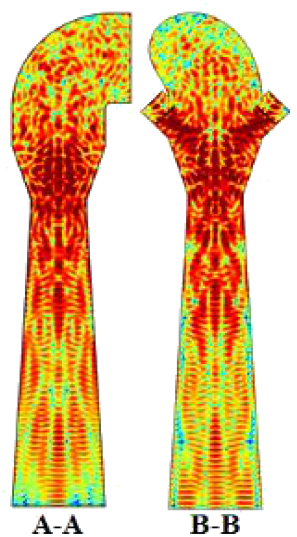

c) $0.14 \mathrm{~m}^{2}$

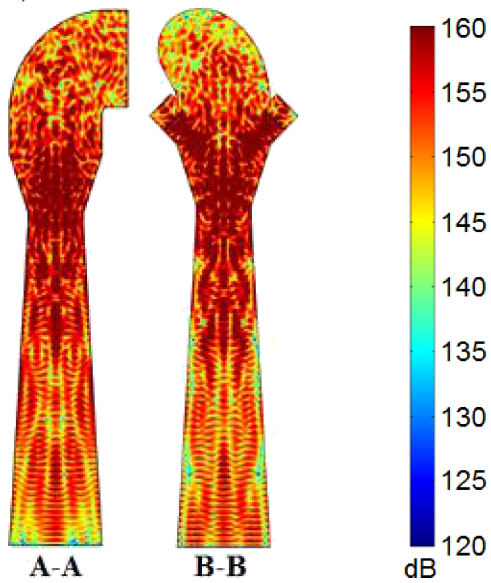

Fig. 22. Distribution of sound pressure for four sources of ultrasonic vibrations of different areas.
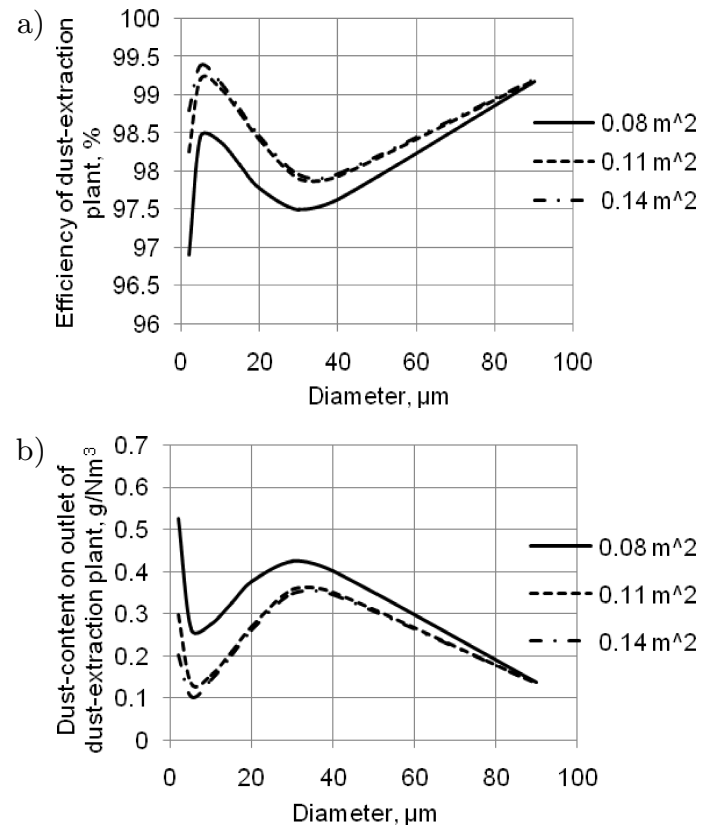

Fig. 23. Dependence of dust extraction plant efficiency (a) and dust content at its output (b) at the application of four radiators of ultrasonic vibrations of various areas. 
a)

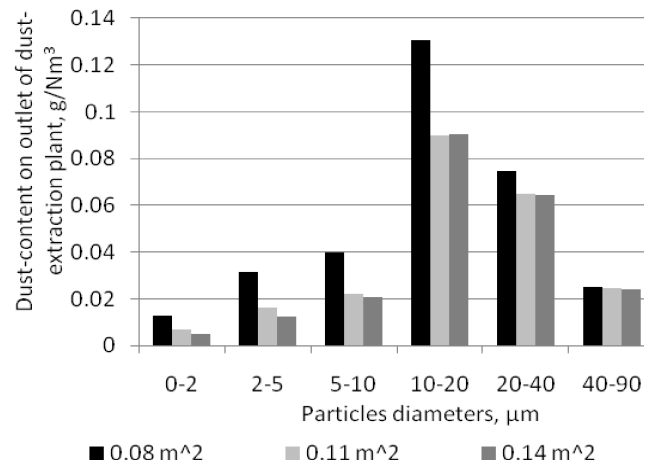

b)

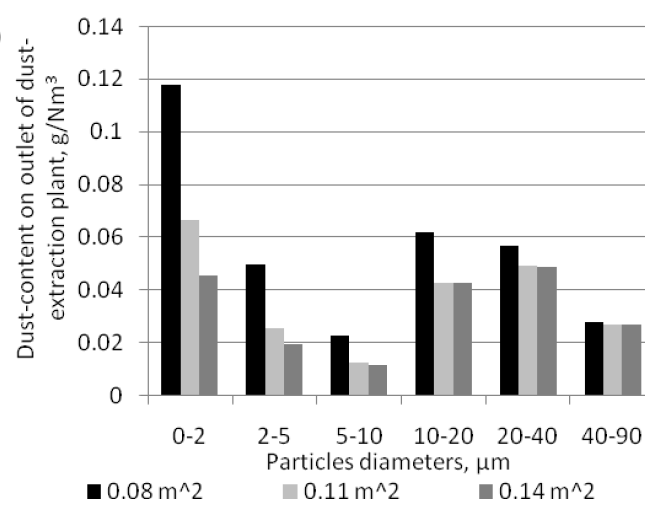

Fig. 24. Fraction ash composition obtained at burning of coal from Kharanor coal deposit (a) and Ekibastuz field (b) at the output of the dust extraction plant at the application of four sources of ultrasonic action of various areas.

a) $0.08 \mathrm{~m}^{2}$

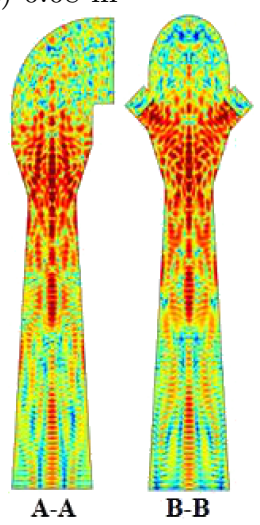

b) $0.11 \mathrm{~m}^{2}$

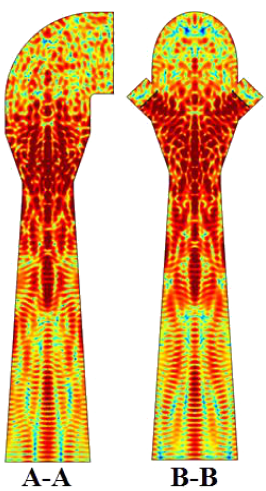

c) $0.14 \mathrm{~m}^{2}$

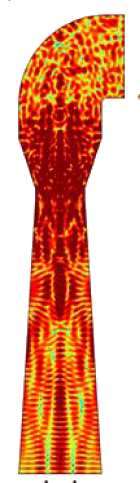

A-A
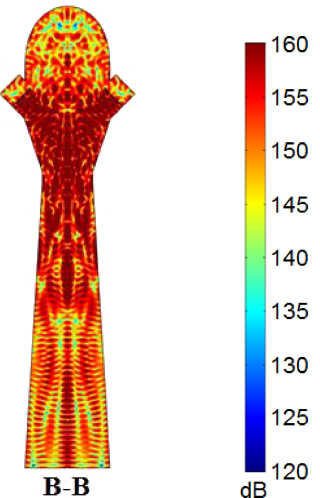

Fig. 25. Distribution of sound pressure level for various radiating surface areas at the installation of six sources of ultrasonic vibrations.
It proves the results of calculations of dust extraction plant efficiency at the use six radiators of ultrasonic vibrations presented in Figs. 26, 27.

a)

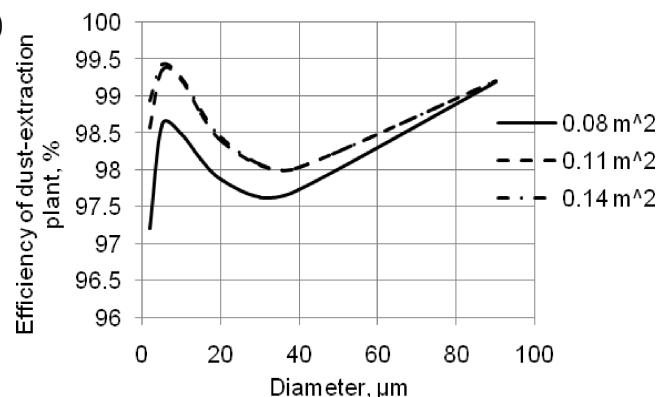

b)

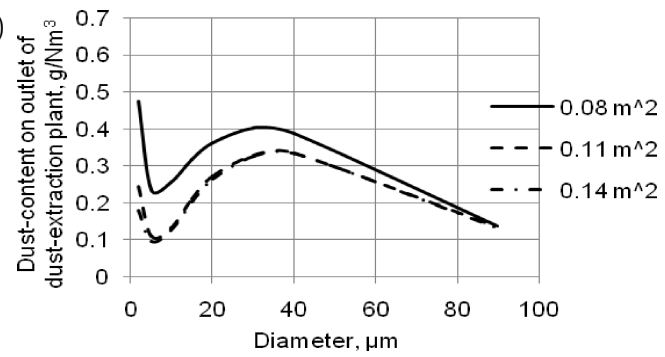

Fig. 26. Dependence of dust extraction plant efficiency (a) and dust content at its output (b) on the size of ash particles at six radiators of ultrasonic vibrations of various areas.

a)

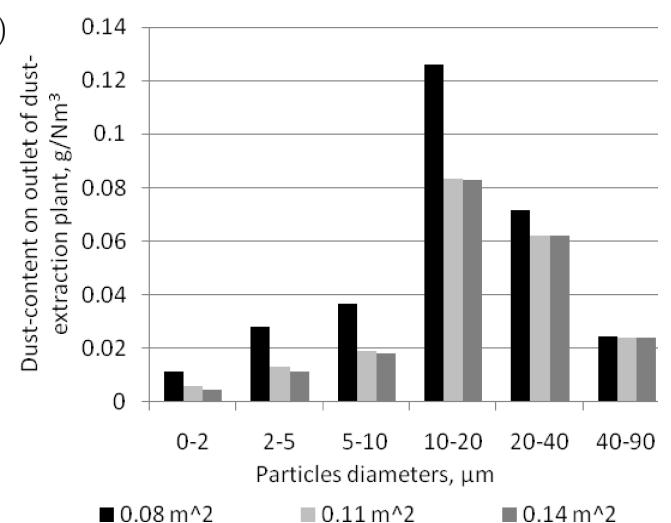

b)

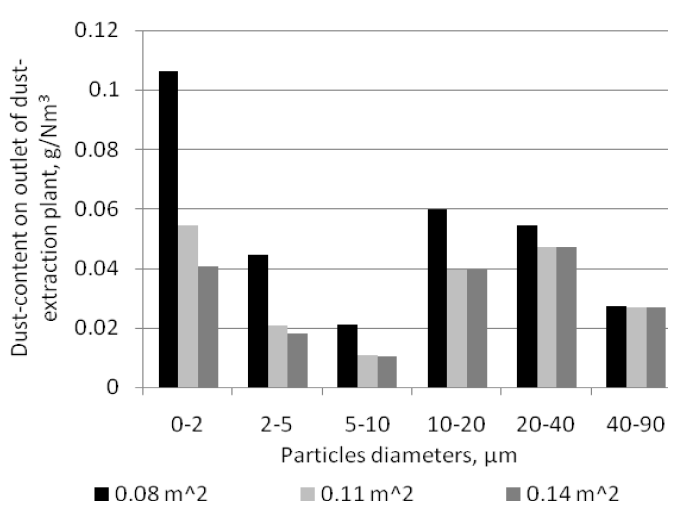

Fig. 27. Fraction ash composition obtained at burning of coal from Kharanor coal deposit (a) and Ekibastuz field (b) at the output of the dust extraction plant at the application of six sources of ultrasonic vibrations of various areas. 
Figure 28 shows dependences of dust content at the output of the dust extraction plant on the radiating surface area at different numbers of the radiators of ultrasonic vibrations.

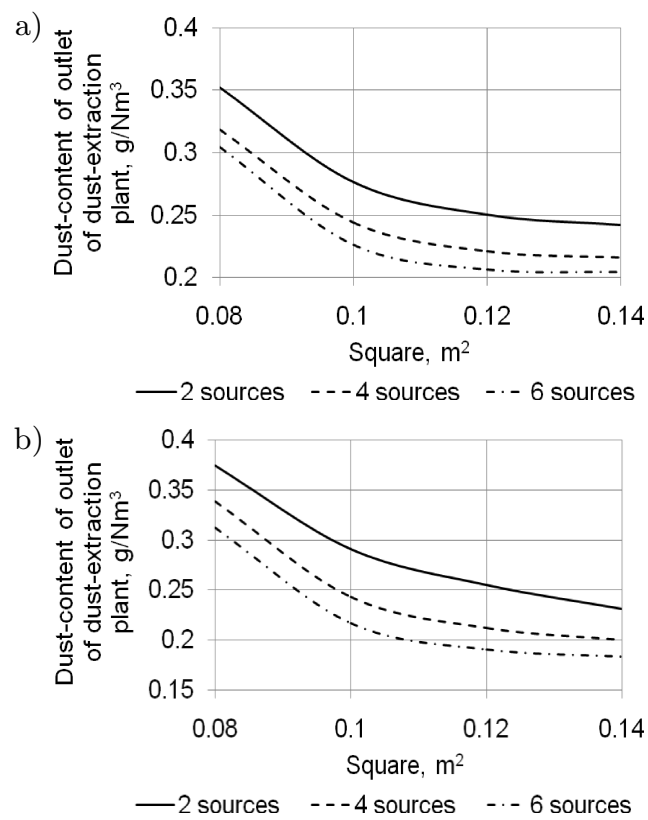

Fig. 28. Dependences of dust content at the output of the dust extraction plant on the radiating surface area at various numbers of radiators of ultrasonic vibrations at burning of coal of Kharanor coal deposit (a) and Ekibastuz field (b).

The analysis of obtained results proves the fact that the application of six sources of ultrasonic vibrations can provide close to required residual dust content at the output of the dust extraction plant (no more than $0.23 \mathrm{~g} / \mathrm{Nm}^{3}$ ) even if radiating surface area is $0.08 \mathrm{~m}^{2}$. Moreover, reachable effect of ultrasonic action on highdispersed ash (less than $10 \mu \mathrm{m}$ ) is considerably higher than the requirements of modern ecological standards.

At that growth of the dust extraction plant (decrease of dust content at the output) reduces beginning with radiating surface area of $0.12 \ldots 0.14 \mathrm{~m}^{2}$ (Fig. 28) and at the application of more than six sources of ultrasonic action.

Thus, the maximum efficiency of the dust extraction plant is provided at the use of the ultrasonic action sources of no more than six pieces with the area in the range of 0.08 to $0.14 \mathrm{~m}^{2}$.

For realization of determined modes of ultrasonic action at the industrial dust extraction plant special ultrasonic radiators, presented further, are proposed, developed and applied.

\section{Practical realization of ultrasonic coagulation in the industrial dust extraction plant}

To prove the efficiency of ultrasonic action and practical realization of ultrasonic coagulation for the

solution of dust extraction problems special apparatuses with the radiators (Fig. 29) having diameter of radiating surface of 320,370 and $418 \mathrm{~mm}$ (with square $0.0804 ; 0.107$ and $0.137 \mathrm{~m}^{2}$, respectively) were developed.

a) 6

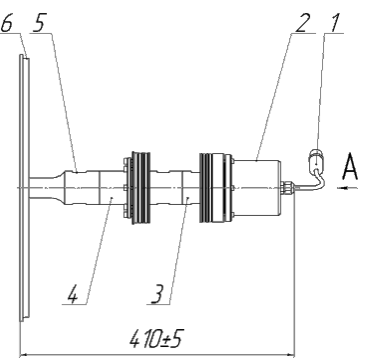

b)

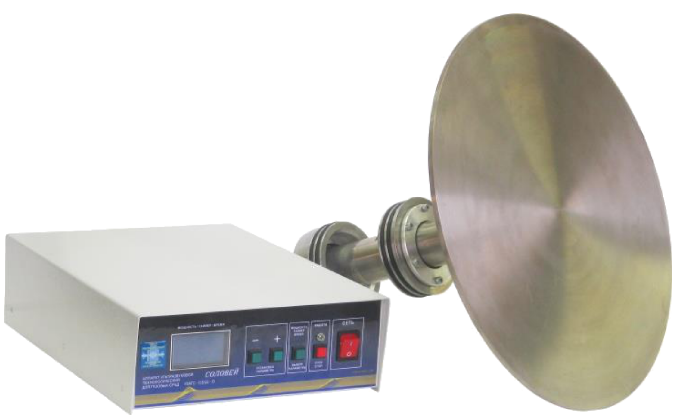

Fig. 29. Construction of the radiator (a) and appearance (b) of the apparatus with the disk operating radiator with the diameter of $418 \mathrm{~mm}$ : 1 - power cable; 2 - piezoelectric transducer; 3 - booster link for cooling; 4 - part of the waveguide; 5 - concentrator; 6 - disk radiator.

The base of the radiators is made of titanium disks of step variable thickness (KHMELEV et al., 2009; 2010a) making flexural vibrations at the frequency of $22 \mathrm{kHz}$, which is multiple $(5,7,9)$ of the basic mode of disk vibrations. The use of flexural vibrations provides increase of energy output (more than $75 \%$ ) into gas medium, radiation from the surface of the parts of various thicknesses ensures coherence of radiated ultrasonic waves (KHMELEV et al., 2009). High efficiency of radiation and absence of reciprocal compensation of waves from different points of the surface of the radiator allow generate vibrations with sound pressure level of $145 \mathrm{~dB}$ at the distances exceeding diameter of the working tool. Special form of the radiating and back surfaces of the disk provides uniformity of radiation. The piezoelectric transducers are used to provide vibrations of the disk radiators, the concentrators are applied for intensification of vibrations (KHMELEV et al., 2010c). Between the transducer and the concentrator there is a booster resonance link providing formation of cooling system of the piezoelectric transducer at the operation of the radiators in the conditions of high temperatures.

The radiator of diameter $418 \mathrm{~mm}$ with the piezoelectric transducer, the concentrator and the booster 
are presented in Fig. 29a. The appearance of the apparatus with such radiator is shown in Fig. $29 \mathrm{~b}$.

Developed radiators were integrated into the construction of the typical dust extraction plant used for dust extraction at the thermal power plant worked at Kharanor coal deposit. Initial particles composition of the fly ash is given in Fig. 29. The radiators were installed into the cap of Venturi tube to avoid their abrasive wear by particle flow.

For testing, 2 radiators of diameter $418 \mathrm{~mm}$ mounted into Venturi tube at an angle of 45 degrees were used. According to theoretical calculations, the number of emitters is the minimum necessary to comply with environmental standards for flue gas dust content at the exit dust-extraction plant (no more than $\left.0.3 \mathrm{~g} / \mathrm{Nm}^{3}\right)$. Comparative data of test results and theoretical calculations are shown in Fig. 30.
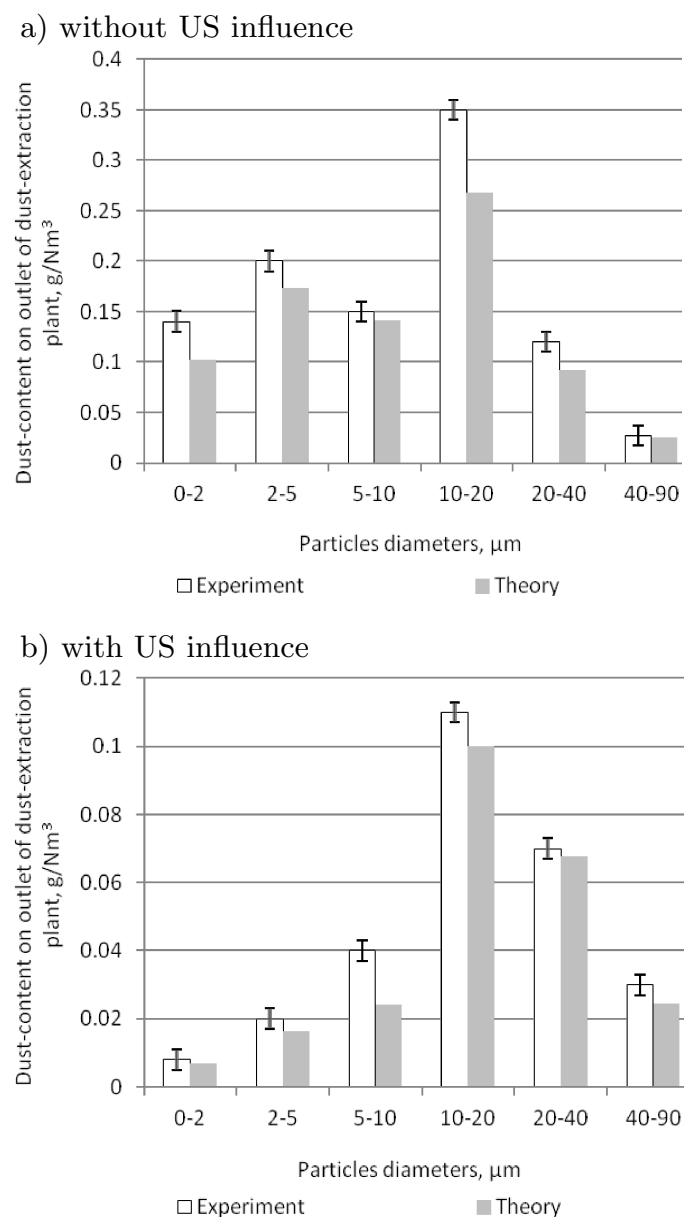

Fig. 30. Dispersed composition on outlet of Venturi tube.

As follows from the presented data, the radiators provide a reduction of dust content at dust-extraction plant output from $17 \mathrm{~g} / \mathrm{Nm}^{3}$ to $0.28 \mathrm{~g} / \mathrm{Nm}^{3}$ (without US influence dust content at output reduced to only $0.98 \mathrm{~g} / \mathrm{Nm}^{3}$ ). This means that ultrasonic impact reduces dust content at plant output in more than 4 times. Thus, on a small fraction (less than $5 \mu \mathrm{m}$ ) of ash, the radiators provide reducing of dust content at plant output up to 15 times or more. This confirms the prospects of application of ultrasonic vibrations to improve the efficiency of wet gas cleaning systems. Comparing the experimental results with theoretical calculations (no more than $0.23 \mathrm{~g} / \mathrm{Nm}^{3}$ ) for radiators $418 \mathrm{~mm}$ shows that the difference does not exceed $18 \%$. This suggests the prospect of application of the method to calculate the efficiency of the ultrasonic vibration in the gas cleaning processes. The difference between the experimental and theoretical data is due to assumptions that the model does not take into account the turbulent fluctuations and asphericity form of ash particles.

\section{Conclusion}

As a result of carried out studies the possibility of efficiency increase of collecting of fine-dispersed particles in Venturi tubes due to the entry of ultrasonic vibrations was demonstrated. We also determined modes and conditions of ultrasonic action (frequency, intensity, number and location of the radiators), developed apparatuses with the radiators of various sizes.

During studies it is stated that:

1. Ultrasonic action with sound pressure level of $150 \mathrm{~dB}$ provides no less than double decrease of dust content at the output of Venturi tube at the extraction of particles with size of $20 \mu \mathrm{m}$ inclusively and in 1.5 times at the collection of particles with the size of more than $20 \mu \mathrm{m}$. At that the most essential efficiency growth of gas cleaning is observed at the extraction of fine-dispersed particles (less than $5 \mu \mathrm{m}$ ). Efficiency of particle extraction with the size of $2 \mu \mathrm{m}$ under ultrasonic action with sound pressure level of $150 \mathrm{~dB}$ increases up to $99 \%$.

2. Optimum for efficient (providing more than fourfold decrease of residual dust content at the output of the dust extraction plant) gas cleaning is the mode of ultrasonic action at the frequency of $22 \mathrm{kHz}$ with sound pressure level of $145 \ldots 155 \mathrm{~dB}$ at the installation of two radiators with area of $0.14 \mathrm{~m}^{2}$, four radiators with area of $0.11 \mathrm{~m}^{2}$ or six radiators with area of $0.08 \mathrm{~m}^{2}$ at the angle of 45 degrees to the axis of Venturi tube.

3. Realization of ultrasonic action is the most efficient for the reduction (up to 15 times) of the content of fine-dispersed fraction ( $2 \mu \mathrm{m}$ and less), which is impossible to extract without ultrasonic action.

\section{Acknowledgment}

The reporter study was financially supported by Russian Foundation for Basic Research (RFBR), project no. 16-38-60082 mol_a_dk. 


\section{References}

1. Balabekov O.S., Baltabaev L.Sh. (1991), Purification of gases in chemical industry. Processes and apparatuses [in Russian], Chemistry, Moscow.

2. Chernov N.N. (2004), Acoustic methods and means of precipitation of suspended particles of industrial flue gases [in Russian], D.Sc. Thesis, Taganrog State Radiotechnical University.

3. Danser H.W., Neumann E.P. (1949), Industrial sonic agglomeration and collection systems, Industr. and Eng. Chem., 41, 11, 2439.

4. Flagan R.C., Seinfeld J.H. (1988), Fundamentals of air pollution engineering, Prentice-Hall, Inc., Englewood Cliffs, New Jersey.

5. Gallego-Juarez J.A., Rodriguez-Corral G., Riera-Franco de Sarabia E., Hoffmann T.L., Calvez-Moraleda J.C. (1999), Application of acoustic agglomeration to reduce fine particle emissions from coal combustion plants, Environmental Science and Technology, 33, 21.

6. Hygienic Standards 2.1.6.695-98 (1998), Maximum admissible concentration of polluting substances in air of residential area [in Russian].

7. Khmelev V.N., Galakhov A.N., Tsyganok S.N., Lebedev A.N., Shalunov A.V., Khmelev M.V. (2010a), Ultrasonic coagulation on the basis of piezoelectric vibrating system with focusing radiator in the form of step-variable plate, 11th Annual International Conference and Seminar on Micro/Nanotechnologies and Electron Devices, pp. 376-379, Novosibirsk.

8. Khmelev V.N., Lebedev A.N., Tsyganok S.N., Shalunov A.V., Galahov A.N., Shalunova K.V. (2009), Multifrequency ultrasonic transducer with stepped-plate disk, Proceedings of 10th International Workshop and Tutorials on Electron Devices and Materials, pp. 250-253, Novosibirsk.

9. Khmelev V.N., Shalunov A.V., Golykh R.N., Shalunova K.V. (2010b), Theoretical study of acoustic coagulation of gas-dispersed systems, Proceedings of 11th Annual International Conference and Seminar on Micro/Nanotechnologies and Electron Devices, pp. 328-333, Novosibirsk.

10. Khmelev V.N., Shalunov A.V., Shalunova K.V. (2008), The acoustical coagulation of aerosols, Proceedings of 9th International Workshop and Tutorials on Electron Devices and Materials Proceedings, Novosibirsk.

11. Khmelev V.N., Shalunov A.V., Shalunova K.V., Shalunova A.V., Antonnikova A.A. (2012), Study of possibility of ultrasonic coagulation in air flow, Proceedings of 13th International Workshop and Tutorials on Electron Devices and Materials, pp. 183-187, Novosibirsk.

12. Khmelev V.N., Shalunov A.V., Shalunova K.V., Tsyganok S.N., Barsukov R.V., Slivin A.N. (2010c), Ultrasonic coagulation of aerosols [in Russian], Publisher of Altay State Technical University, Barnaul.

13. Kouzov P.A., Malgin A.D., Skryabin G.M. (1993), Purification of gases and air form dust in chemical industry [in Russian], Chemistry, Leningrad.

14. Kropp L.I., Akbrut A.I. (1977), Dust-collectors with Venturi tubes at thermal power stations, Energy [in Russian], Moscow.

15. Kudryashova O.B., Antonnikova A.A., Titov S.S. (2013), Physical and mathematical model of the coagulation of micron and submicron aerosols with regard for evaporation and sedimentation at ultrasonic effect, Thermophysics and Aeromechanics, 20, 3, 381-384.

16. Lavely L.L., Ferguson A.W. (1996), Power Plant Atmospheric Emissions Control, Power Plant Engineering, pp. 418-463.

17. Mohamed K.K. [Ed.] (2011), The Impact of Air Pollution on Health, Economy, Environment and Agricultural Sources, Publisher InTech, Rijeka.

18. National Ambient Air Quality Standards, United States Environmental Protection Agency.

19. Shalimo M.A. (1965), Acoustic coagulation of cement mixes, Journal of engineering physics, 8, 3, 253-255.

20. Shilyaev M.I., Shilyaev A.M., Grischenko E.P. (2006), Methods for calculation of dust collectors [in Russian], Publisher of Tomsk State University of Architecture and Civil Engineering.

21. Shtokman E.A. (1977), Purification of air from dust at the enterprises of food industry [in Russian], Food industry, Moscow.

22. Shtokman E.A. (1999), Air purification [in Russian], ASV publishing, Moscow.

23. Skryabina L.Y. (1980), Industrial and sanitary purification of gases. Atlas of industrial dusts. Part $I$. Flue ash of the thermal power stations, Central Institute of Scientific-Technical Information and TechnicalEconomical Studies of Chemical and Oil Mechanical Engineering, Moscow.

24. Sommerfeld M. (2001), Validation of a stochastic Lagrangian modelling approach for inter-particle collisions in homogeneous isotropic turbulence, Int. J. of Multiphase Flow, 27, 1829-1858.

25. St. Clair H.W. (1949), Agglomeration of smoke, fog or dust particles by sonic waves, Industr. and Eng. Chem., 41, 11, 2434.

26. Uzhov V.N. (1981), Cleaning of industrial gases from dust [in Russian], Chemistry, Moscow.

27. Vincent B. [Ed.] (1987), Coagulation Kinetics and Structure Formation, Publisher Springer, US.

28. Zhang G.X., Liu J.Zh., WAng J., Zhou J.H., CEN K.F. (2012), Numerical simulation of acoustic wake effect in acoustic agglomeration under Oseen flow condition, Chinese Science Bulletin, 57, 19. 